\title{
Evidence for Existence of Real Causality in the Case of Zero Granger Causality
}

\author{
Sanqing Hu, Yu Cao, Jianhai Zhang, Wanzeng Kong, Kun Yang, Xun Li, and Yanbin Zhang
}

\begin{abstract}
Granger causality is one of the most popular measures to reveal causality influence of time series and has been widely applied in economics and neuroscience due to its simplicity and easy implementation. In this paper, we first introduce two key assumptions which are showed to be prerequired for defining Granger causality; that is, without the two assumptions, one cannot draw a conclusion that there is no real causality even if we derive zero Granger causality. We then discuss two cases for which Granger causality is zero but there exists real causality between two time series. Therefore, researchers must be caution in drawing any conclusion based on Granger causality value.
\end{abstract}

\section{INTRODUCTION}

Given a set of time series, how to define causality influence among them has been a topic for over two thousand years and has yet to be completely resolved. In the literature one of the most popular definitions for causality is Granger causality (Granger causality). Due to its simplicity and easy implementation, Granger causality has been widely used. The basic idea of Granger causality was originally conceived by Wiener [47] and later formalized by Granger in the form of linear regression model [20]. It can be simply stated as follows: if the variance $\left(\sigma_{\epsilon_{1}}^{2}\right)$ of the prediction error for the first time series at the present time is not less than the variance $\left(\sigma_{\eta_{1}}^{2}\right)$ of the prediction error by including past measurements from the second time series in the linear regression model, then the second time series can be said to have a causal (driving) influence on the first time series. Reversing the roles of the two time series one repeats the process to address the question of driving in the opposite direction. Granger causality value of $\ln \left(\sigma_{\epsilon_{1}}^{2} / \sigma_{\eta_{1}}^{2}\right)$ is defined to describe the strength of the causality which the second time series has on the first one [13]-[15], [20], [22], [35]. From Granger causality value, it is clear that (i) $\ln \left(\sigma_{\epsilon_{1}}^{2} / \sigma_{\eta_{1}}^{2}\right)=0$ when there is no causal influence from the second time series to the first one and $\ln \left(\sigma_{\epsilon_{1}}^{2} / \sigma_{\eta_{1}}^{2}\right)>0$ when there is; (ii) the larger the value of $\ln \left(\sigma_{\epsilon_{1}}^{2} / \sigma_{\eta_{1}}^{2}\right)$, the higher causal influence. In recent years there has been significant interest to discuss causal interactions between brain areas which are highly complex neural networks. For instance, with Granger causality analysis Freiwald et al. [14] revealed the

Sanqing $\mathrm{Hu}$, Jianhai Zhang, Wanzeng Kong, Kun Yang, Xun $\mathrm{Li}$, and Yanbin Zhang are with College of Computer Science, Hangzhou Dianzi University, Hangzhou, Zhejiang, China (email: \{sqhu, jhzhang,kongwanzeng,yangkun,xunli,zhyb \}@hdu.edu.cn). Yu Cao is with College of Engineering and Computer Science, The University of Tennessee at Chattanooga, Chattanooga, TN 37403 USA (email: yu-cao@utc.edu ).

The work was supported by Natural Science Foundation of China (No. 61070127), International Cooperation Project of Zhejiang Province, China (No. 2009C14013) existence of both uni- and bi-directional influences between neural groups in the macaque inferotemporal cortex. Hesse et al. [22] analyzed EEG data from the Stroop task and disclosed that conflict situations generate dense webs of interactions directed from posterior to anterior cortical sites and the web of directed interactions occurs mainly $400 \mathrm{~ms}$ after the stimulus onset and lasts up to the end of the task. Roebroeck et al. [38] explored directed causal influences between neuronal populations in fMRI data. Oya et al. [35] demonstrated causal interactions between auditory cortical fields in humans through intracranial evoked potentials to sound. Gow et al. [19] applied Granger analysis to MRI constrained MEG and EEG data to explore the influence of lexical representation on the perception of ambiguous speech sounds. Gow et al. [18] showed a consistent pattern of direct posterior superior temporal gyrus influence over sites distributed over the entire ventral pathway for words, non-words, and phonetically ambiguous items.

In this paper, we point out that Granger causality in time domain cannot correctly reveal real causality between two time series in some cases. To do so, we introduce two obvious key assumptions for defining Granger causality. The two assumptions are based on the two estimated models (autoregression model and joint regression model) of two time series and require checking re-estimation problem of realizations of the estimated joint regression model. Only when the two assumptions are satisfied, zero Granger causality does mean no real causality. Without the two assumptions, one cannot draw a conclusion that there is no real causality even if we derive zero Granger causality. Therefore, checking re-estimation problem of realizations of the estimated joint regression model is very important when researchers obtain zero Granger causality in the study of time series data. We then gave two cases for which Granger causality is zero but there exists real causality between two time series. Therefore, researchers must be caution in drawing any conclusion based on Granger causality value in time domain.

This paper is organized as follows. Causality analysis in time domain and frequency domain are discussed in Section II and Section III respectively. Several examples are provided in Section IV. Concluding remarks are given in Section V. The rest of this paper is organized as follows: Section II derives an equivalent linear programming (LP) formulation of KWTA, which is suitable for the neural network design. Section III introduces the neural network design procedure, architecture and properties. Some simulation results are presented in Section IV to show its performance. Finally, Section $\mathrm{V}$ concludes the paper. 


\section{GRANGER CAUSALITY}

In this section we first introduce the well-known Granger causality and conditional Granger causality. We begin with bivariate time series. Given two stochastic process $X_{1}(t)$ and $X_{2}(t)$ which are assumed to be jointly stationary. Their autoregresive representations are described as

$$
\left\{\begin{aligned}
X_{1, t} & =\sum_{j=1}^{m} \mathbf{a}_{11, j} X_{1, t-j}+\epsilon_{1, t} \\
X_{2, t} & =\sum_{j=1}^{m} \mathbf{a}_{22, j} X_{2, t-j}+\epsilon_{2, t}
\end{aligned}\right.
$$

and their joint representations are described as

$$
\left\{\begin{array}{l}
X_{1, t}=\sum_{j=1}^{m} a_{11, j} X_{1, t-j}+\sum_{j=1}^{m} a_{12, j} X_{2, t-j}+\eta_{1, t} \\
X_{2, t}=\sum_{j=1}^{m} a_{21, j} X_{1, t-j}+\sum_{j=1}^{m} a_{22, j} X_{2, t-j}+\eta_{2, t}
\end{array}\right.
$$

where $t=0,1, \cdots, N$, the noise terms are uncorrelated over time, $\epsilon_{i}$ and $\eta_{i}$ have zero means and variances of $\sigma_{\epsilon_{i}}^{2}$ and $\sigma_{\eta_{i}}^{2}, i=1,2$. The covariance between $\eta_{1}$ and $\eta_{2}$ is defined by $\sigma_{\eta_{1} \eta_{2}}=\operatorname{cov}\left(\eta_{1}, \eta_{2}\right)$.

Now consider the first equalities in (1) and (2). According to the original formulations in [20] and [47], if $\sigma_{\eta_{1}}^{2}$ is less than $\sigma_{\epsilon_{1}}^{2}$ in some suitable statistical sense, then $X_{2}$ is said to have a causal influence on $X_{1}$. In this case, the first equality in (2) is more accurate than that in (1) to estimate $X_{1}$. Otherwise, if $\sigma_{\eta_{1}}^{2}=\sigma_{\epsilon_{1}}^{2}$, then $X_{2}$ is said to have no causal influence on $X_{1}$. In this case, the two equalities are almost same. Such kind of causal influence called Granger causality (Granger causality) [13], [15], can be defined by

$$
F_{X_{2} \rightarrow X_{1}}=\ln \frac{\sigma_{\epsilon_{1}}^{2}}{\sigma_{\eta_{1}}^{2}} \text {. }
$$

Obviously, $F_{X_{2} \rightarrow X_{1}}=0$ when there is no causal influence from $X_{2}$ to $X_{1}$ and $F_{X_{2} \rightarrow X_{1}}>0$ when there is. Similarly, The causal influence from $X_{1}$ to $X_{2}$ is defined by

$$
F_{X_{1} \rightarrow X_{2}}=\ln \frac{\sigma_{\epsilon_{2}}^{2}}{\sigma_{\eta_{2}}^{2}} .
$$

To show whether the interaction between two time series is direct or is mediated by another recorded time series, conditional Granger causality [13], [16], was defined by

$$
F_{X_{2} \rightarrow X_{1} \mid X_{3}}=\ln \frac{\sigma_{\epsilon_{3}}^{2}}{\sigma_{\eta_{3}}^{2}}
$$

where $\sigma_{\epsilon_{3}}^{2}$ and $\sigma_{\eta_{3}}^{2}$ are variances of two noise terms, $\epsilon_{3}$ and $\eta_{3}$, of the following two joint autoregressive representations:

$$
X_{1, t}=\sum_{j=1}^{m} \mathbf{a}_{11, j} X_{1, t-j}+\sum_{j=1}^{m} \mathbf{a}_{13, j} X_{3, t-j}+\epsilon_{3, t}
$$

and

$$
\begin{aligned}
X_{1, t}= & \sum_{j=1}^{m} a_{11, j} X_{1, t-j}+\sum_{j=1}^{m} a_{12, j} X_{2, t-j} \\
& +\sum_{j=1}^{m} a_{13, j} X_{3, t-j}+\eta_{3, t} .
\end{aligned}
$$

According to this definition, $F_{X_{2} \rightarrow X_{1} \mid X_{3}}=0$ means that no further improvement in the prediction of $X_{1}$ can be expected by including past measurements of $X_{2}$. On the other hand, when there is still a direct component from $X_{2}$ to $X_{1}$, the past measurements of $X_{1}, X_{2}, X_{3}$ together result in better prediction of $X_{1}$, leading to $\sigma_{\eta_{3}}^{2}<\sigma_{\epsilon_{3}}^{2}$, and $F_{X_{2} \rightarrow X_{1} \mid X_{3}}>0$.

In above Granger causality and conditional Granger causality, both of them emphasize that zero causality comes from no further improvement in the prediction of $X_{1}$ by including past measurements of $X_{2}$. As such, in his early work [15] Geweke stated that Granger causality $F_{X_{2} \rightarrow X_{1}}=$ $0 \Leftrightarrow \sigma_{\epsilon_{1}}^{2}=\sigma_{\eta_{1}}^{2} \Leftrightarrow a_{12, j} \equiv 0$. According to this statement, if $a_{12, j} \equiv 0$, then there is no causality (or Granger causality). If $a_{12, j} \not \equiv 0$, then there is Granger causality. In this paper, we will focus on Granger causality (similar discussions for conditional Granger causality) and show that Granger causality being zero does not necessarily mean no real causality between two time series, in other words, there may still exist real causality between two time series in the case of zero Granger causality. To do so, we point out the following two obvious key assumptions for defining Granger causality from $X_{2}$ to $X_{1}$.

Assumption 1: After we obtain two estimated Model (1) and Model (2) for two given time series, for any realization of the estimated Model (2) we estimate again and get the same Model (1) and Model (2) as before, that is, all coefficients keep unchanged.

Assumption 2: If $a_{12, j} \equiv 0$, then in the estimated Model (1) (or Model (2) in this case), $X_{1, t-j}$ has nothing to do with $X_{2}$ 's past values.

Only in above assumptions, we can say $F_{X_{2} \rightarrow X_{1}}=0 \Leftrightarrow$ $a_{12, j} \equiv 0$; that is, to determine whether or not there is causal influence from $X_{2}$ to $X_{1}$ we only need to check whether or not $a_{12, j} \equiv 0$. If $a_{12, j} \equiv 0$, then surely we can have $\sigma_{\epsilon_{1}}^{2}=\sigma_{\eta_{1}}^{2}$ resulting in $F_{X_{2} \rightarrow X_{1}}=0$. As usual, all researchers directly calculate Granger causality based on the two estimated Model (1) and Model (2) for two given time series and do not check re-estimation problem of realizations of the estimated Model (2) at all. In the following, we will show that the situation may change without above assumptions. That is, without above assumptions, Granger causality $F_{X_{2} \rightarrow X_{1}}=0$ does not necessarily mean $a_{12, j} \equiv 0$ and thus there exists real causality from $X_{2}$ to $X_{1}$.

\section{Two CASES For ZERo Granger CAUSALITy}

In this section we will analyze the following two cases for Model (2): Case A. $\eta_{2, t} \equiv 0$. Case B. $\eta_{1, t}=\eta_{2, t}$. We will show $F_{X_{2} \rightarrow X_{1}}=0$ in these two cases and there exist real causalities for both of two cases. To do so, let's introduce spectral Granger causality as follows.

Spectral Granger causality ([8], [12], [13], [15], [16]): Given the bivariate Model (2), the Granger casual influence from $X_{2}$ to $X_{1}$ is defined by

$$
\begin{gathered}
I_{X_{2} \rightarrow X_{1}}(f)=-\log \left(1-\frac{\left(\sigma_{\eta_{2}}^{2}-\frac{\sigma_{\eta_{1} \eta_{2}}^{2}}{\sigma_{\eta_{1}}^{2}}\right)\left|H_{12}(f)\right|^{2}}{S_{X_{1} X_{1}}}\right) \\
\in[0,+\infty)
\end{gathered}
$$


where the transfer function is $H(f)=A^{-1}(f)$ whose components are

$$
\begin{gathered}
H_{11}(f)=\frac{1}{\operatorname{det}(A)} \bar{a}_{22}(f), H_{12}(f)=-\frac{1}{\operatorname{det}(A)} \bar{a}_{12}(f), \\
H_{21}(f)=-\frac{1}{\operatorname{det}(A)} \bar{a}_{21}(f), H_{22}(f)=\frac{1}{\operatorname{det}(A)} \bar{a}_{11}(f), \\
A=\left[\bar{a}_{i j}\right]_{2 \times 2}, \bar{a}_{k k}(f)=1-\sum_{j=1}^{m} a_{k k, j} e^{-i 2 \pi f j}, k=1,2, \\
\bar{a}_{h l}(f)=-\sum_{j=1}^{m} a_{h l, j} e^{-i 2 \pi f j}, h, l=1,2, h \neq l .
\end{gathered}
$$

For the above two cases and for any coefficients $a_{11, j}, a_{12, j}, a_{21, j}, a_{22, j}$, we can check $I_{X_{2} \rightarrow X_{1}}(f) \equiv 0$ based on (8). On the other hand, noting the relationship (17.29) in [13] between time and frequency domains, we can easily see Granger causality $F_{X_{2} \rightarrow X_{1}}=0$ for both of Case A and Case $B$, that is, no Granger causality. Now one question is arising: Does there exist real causality for Case A or Case B? the answer is Yes. Let's discuss each case in detail as follows.

Case A. $\eta_{2, t} \equiv 0$. Let's consider the following very simple model:

$$
\left\{\begin{array}{l}
X_{1, t}=-0.99 X_{2, t-1}+\eta_{1, t} \\
X_{2, t}=-X_{2, t-2}
\end{array}\right.
$$

where $\eta_{1}$ is a white noise process with zero mean and variance $\sigma_{\eta_{1}}^{2}=1$. Since $\eta_{2, t} \equiv 0$ we have $F_{X_{2} \rightarrow X_{1}}=$ 0 . No Granger causality! However, by observing the first equality of (10), real causal influence from $X_{2}$ to $X_{1}$ indeed exists. Moreover, for one realization (called realization A) of Model (10) with $X_{2}$ 's initial conditions being $X_{2,1}=$ $6.0869, X_{2,2}=-1.3372$, we plot trajectories $X_{1, t}$ and $\eta_{1, t}$ in Figure 1A and 1B from which one can clearly see that $X_{1}$ has larger amplitudes compared with $\eta_{1}$. So, what causes such kind of larger amplitudes for $X_{1}$ ? Of course, it is $X_{2}$ because there is no $X_{1}$ 's past value at all in Model (10). For another realization (called realization B) of Model (10) with $X_{2}$ 's initial conditions being $X_{2,1}=X_{2,2}=0$ and $\eta_{1, t}$ being same as in realization $\mathrm{A}$, we can see $X_{1, t}=\eta_{1, t}$ in this case. So, what causes the big change of amplitudes of $X_{1}$ from realization $\mathrm{A}$ to realization $\mathrm{B}$ ? Of course, it is due to the change of $X_{2}$ (i.e, the change of the initial conditions $X_{2,1}$ and $X_{2,2}$ ). Realization A and realization B further support the existence of causal influence from $X_{2}$ to $X_{1}$. Now one may ask: now that there exists real causal influence from $X_{2}$ to $X_{1}$, why we get Granger causality $F_{X_{2} \rightarrow X_{1}}=0$ ? The reason is as follows: for any realization of Model (10) when we estimate autoregression model, we get

$$
\left\{\begin{array}{l}
X_{1, t}=\sum_{j=1}^{m} a_{j} X_{1, t-j}+\epsilon_{1, t} \\
X_{2, t}=-X_{2, t-2}
\end{array}\right.
$$

where $\sigma_{\epsilon_{1}}^{2}=\sigma_{\eta_{1}}^{2}=1$ (resulting in $F_{X_{2} \rightarrow X_{1}}=\ln \frac{\sigma_{\epsilon_{1}}^{2}}{\sigma_{\eta_{1}}^{2}}=0$ ), which means no any improvement when using the joint regression Model (10). Moreover, for different realization, one gets different coefficient $a_{j}$, which means that $a_{j}$ is related to initial conditions of $X_{2}$. Now we rewrite Model (11) as

$$
\left\{\begin{array}{l}
X_{1, t}=\sum_{j=1}^{m} a_{j} X_{1, t-j}+0 \times X_{2, t-1}+\epsilon_{1, t} \\
X_{2, t}=-X_{2, t-2} .
\end{array}\right.
$$

Could we say $X_{2}$ has no causal influence on $X_{1}$ based on Model (12) because the coefficient of $X_{2}$ in the first equality is zero? No, absolutely no. Note that $a_{j}$ keep changed for different realizations. So, above Assumption 1 is violated and Model (12) is different from Model (2) which satisfies the assumption and then is used to define Granger causality. Hence, from another aspect, Model (12) further confirms the fact that there exists causal influence from $X_{2}$ to $X_{1}$.

A

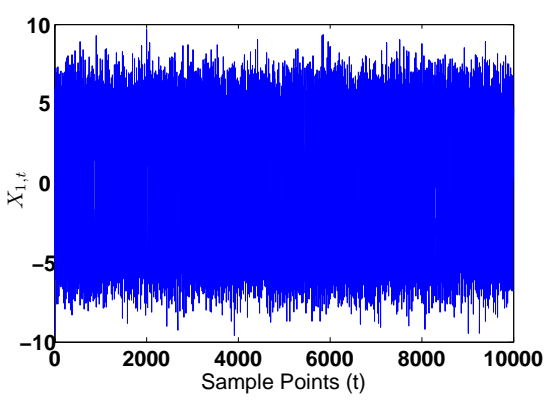

B

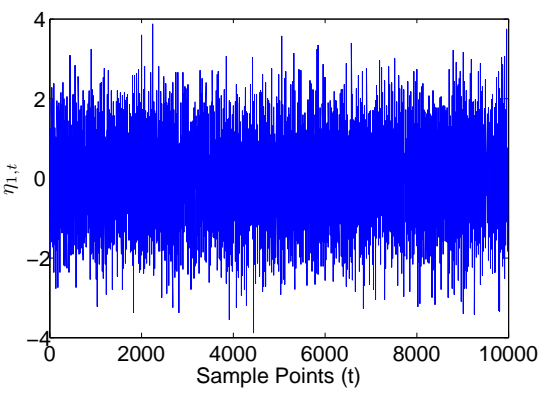

Fig. 1. A) Trajectory $X_{1, t}$ of one realization of above Model (10). B) Trajectory $\eta_{1, t}$ of the same realization as in A).

Case B. $\eta_{1, t}=\eta_{2, t}$.

Let's consider the following very simple model:

$$
\left\{\begin{array}{l}
X_{1, t}=-0.99 X_{2, t-1}+\eta_{1, t} \\
X_{2, t}=\eta_{1, t}
\end{array}\right.
$$

where $\eta_{1}$ is a white noise process with zero mean and variance $\sigma_{\eta_{1}}^{2}=1$. Since $\eta_{2, t}=\eta_{1, t}$ one can obtain Granger causality $F_{X_{2} \rightarrow X_{1}}=0$. No Granger causality! However, if we rewrite Model (13) as

$$
\left\{\begin{array}{l}
X_{1, t}=-0.99 X_{2, t-1}+X_{2, t} \\
X_{2, t}=\eta_{1, t}
\end{array}\right.
$$

then one can clearly see that $X_{1}$ is completely determined by $X_{2}$; that is, $X_{1, t}$ is determined by two parts: $-0.99 X_{2, t-1}$ and $X_{2, t}$. The first part is called to have causal influence on $X_{1}$ from $X_{2}$ 's past value. The second is called to 
have instantaneous causal (see the concept of instantaneous causality in [13]) influence on $X_{1}$ from $X_{2}$ 's current value. Usually, causal influence from $X_{2}$ to $X_{1}$ is talking about the first kind of causal influence. Thus, real causal influence from $X_{2}$ (i.e., $X_{2}$ 's past value) to $X_{1}$ indeed exists by noting that $X_{2}$ does carry predictive information about $X_{1}$ (i.e., the term $\left.0.99 X_{2, t-1}\right)$. On the other hand, in the following, from other aspects, we will show the existence of causal influence from $X_{2}$ to $X_{1}$. For one realization of Model (13) we plot trajectories $X_{1, t}$ and $\eta_{1, t}$ in Figure 2A and 2B from which one can clearly see that $X_{1}$ has larger amplitudes compared with $\eta_{1}$. What causes such kind of larger amplitudes for $X_{1}$ ?

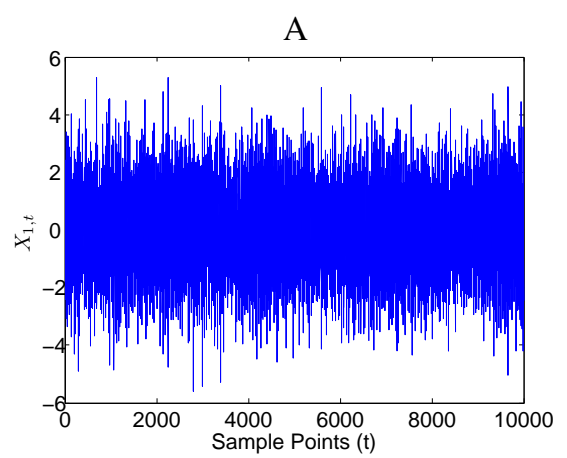

B

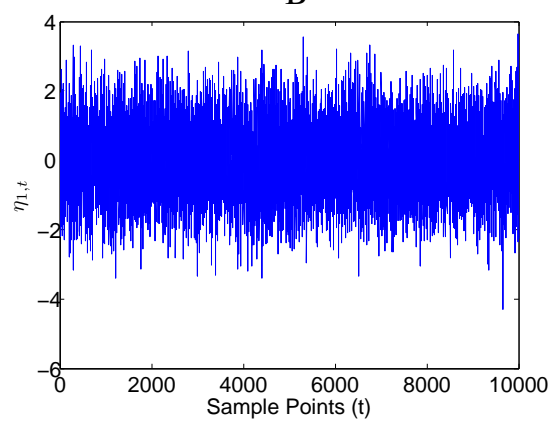

Fig. 2. A) Trajectory $X_{1, t}$ of one realization of above Model (13). B) Trajectory $\eta_{1, t}$ of the same realization as in A).

Of course, it is $X_{2}$ because there is no $X_{1}$ 's past value at all in Model (13). Now one question is arising: now that there exists real causal influence from $X_{2}$ to $X_{1}$, why we get Granger causality $F_{X_{2} \rightarrow X_{1}}=0$ ? The reason is as follows: for any realization of Model (13) when we estimate autoregression model for $X_{1}$, we get

$$
X_{1, t}=\sum_{j=1}^{m} a_{j} X_{1, t-j}+\epsilon_{1, t}
$$

where $\sigma_{\epsilon_{1}}^{2}=\sigma_{\eta_{1}}^{2}=1$ (resulting in $F_{X_{2} \rightarrow X_{1}}=\ln \frac{\sigma_{\epsilon_{1}}^{2}}{\sigma_{\eta_{1}}^{2}}=$ 0 ), which means no any improvement when using the joint regression Model (13). Now we rewrite Model (15) as

$$
X_{1, t}=\sum_{j=1}^{m} a_{j} X_{1, t-j}+0 \times X_{2, t-1}+\epsilon_{1, t} \text {. }
$$

Could we say $X_{2}$ has no causal influence on $X_{1}$ based on Model (16) because the coefficient of $X_{2}$ is zero? No, absolutely no. Note that $X_{1, t-j}$ at the right hand side of Model (16) is closely related to $X_{2}$ 's past values based on the relation in (13). Assumption 2 is violated. Thus, Model (16) is different from the first equality in Model (2). This further confirms the fact that there exists real causal influence from $X_{2}$ to $X_{1}$ for Model (13).

\section{Discussions}

In preceding section, we gave two cases under which Granger causality is zero, but real causality may still exist. The main reason lies in the fact that both of the two cases violate Assumptions 1 and 2 respectively. This again confirms that Assumptions 1 and 2 are pre-required for introducing Granger causality. Otherwise, without the two assumptions, Granger causality may lead to misinterpretation results. Therefore, when researchers conduct Granger causality analysis for time series in economics and neuroscience, the first step is to check whether or not the estimated Model (1) and Model (2) satisfy the two assumptions. Only when the two assumptions are satisfied, then one can use Granger causality to reveal whether or not there is causality influence between two series. If one does not check the assumptions, one cannot make a judgement to say there is no real causality between two time series in the case of zero Granger causality.

\section{Conclusions}

In human cognitive neuroscience, researchers are now not limited to only the study of the putative functions of particular brain regions, but moving toward how different brain regions (such as visual cortex, parietal or frontal regions, amygdala, or hippocampus) may causally influence on each other. These causal influences with different strengths and directionalities may reflect the changing functional demands on the network to support cognitive and perceptual tasks. Granger causality, one of the most popular causality measures, was initially widely used in economics and has recently received growing attention in neuroscience to reveal causal interactions for neurophysiological data. Especially in frequency domain several variant forms of Granger causality have been developed to study causal influences for neurophysiological data in different frequency decompositions during cognitive and perceptual tasks.

In our previous work [25] we proposed new spectral causality, pointed out shortcomings and/or limitations of spectral Granger causality, and showed advantages our new spectral causality over spectral Granger causality. In this paper, we discussed Granger causality in time domain. We first mentioned two assumptions which are showed to be prerequired for defining Granger causality; that is, without the two assumptions, one cannot draw a conclusion that there is no real causality even if we derive zero Granger causality. We then gave two cases for which Granger causality is zero but there exists real causality between two time series. Therefore, researchers must be caution in drawing any conclusion based on Granger causality value in time and frequency domains. 


\section{REFERENCES}

[1] H. Akaike, "A new look at the statisticalmodel identification," IEEE Transactions on Automatic Control, vol. 19, no. 6, pp. 716-723, Dec. 1974.

[2] C. Allefeld, P. B Graben, and J. Kurths, Advanced Methods of Electrophysiological Signal Analysis and Symbol Grounding, pp. 276296, 2008.

[3] K. L. Anderson, R. Rajagovindan, G. A. Ghacibeh, K. J. Meador, and M. Ding, "Theta oscillations mediate interaction between prefrontal cortex and medial temporal lobe in human memory," Cerebral Cortex, vol. 20, no. 7, pp. 1604-1612, Jul. 2010.

[4] L. A. Baccal and K. Sameshima, "Partial directed coherence: A new concept in neural structure determination," Biol Cybern, vol. 84, no. 6, pp. 463-474, Jun. 2001.

[5] L. A. Baccal, K. Sameshima, and D. Y. Takahashi, "Generalized Partial Directed Coherence," in Proc. of the 15th International Conference on Digital Signal Processing (DSP2007), 2007, pp. 162-166.

[6] L. A. Baccal, Y. D. Takahashi, and K. Sameshima, "Computer intensive testing for the influence between time series," in Handbook of Time Series Analysis, Berlin: Wiley VCH, 2006, pp. 365-388.

[7] C. Bernasconi and P. Konig, "On the directionality of cortical interactions studied by structural analysis of electrophysiological recordings," Biological Cybernetics, vol. 81, no. 3, pp. 199-210, Sep. 1999.

[8] K. J. Blinowska, R. Kus, and M. Kaminski, "Granger causality and information flow in multivariate processes," Phys Rev E Stat Nonlinear Soft Matter Phys, Part 1, vol. 70, no. 5, pp. 050902, Nov. 2004.

[9] A. Brovelli, M. Ding, A. Ledberg, Y. Chen, R. Nakamura, and S. L. Bressler, "Beta oscillations in a large-scale sensorimotor cortical network: Directional influences revealed by Granger causality," in Proc. of the National Academy of Sciences of the United States of America, vol. 101, no. 26, pp. 9849-9854, 2004

[10] G. Buzsaki, "Theta rhythm of navigation: link between path integration and landmark navigation, episodic and semantic memory," Hippocampus, vol. 15, no. 7, pp. 827-840, 2005.

[11] W. A. Chaovalitwongse, Y. Fan, and R. C. Sachdeo, "On the time series K-nearest neighbor classification of abnormal brain activity," IEEE Trans. on Systems, Man, and Cybernetics-Part A, vol. 37, no. 6, pp. 1005-1016, Nov. 2007.

[12] J. Cui, L. Xu, S. L. Bressler, M. Ding, and H. Liang, "BSMART: a Matlab/C measurebox for analysis of multichannel neural time series," Neural Networks, Special Issue on Neuroinformatics, vol. 21, no. 8, pp. 1094-1104, Oct. 2008

[13] M. Ding, Y. Chen, and S. L. Bressler, "Granger causality: Basic theory and applications to neuroscience," in B. Schelter, M. Winterhalder, \& J. Timmer (Eds.), Handbook of Time Series Analysis. Weinheim: Wiley-VCH, pp. 437-460, 2006.

[14] W. A. Freiwald, P. Valdes, J. Bosch, R. Biscay, J. C. Jimenez, L. M. Rodriguez, V. Rodriguez, A. K. Kreiter, and W. Singer, "Testing nonlinearity and directedness of interactions between neural groups in the macaque inferotemporal cortex," J. of Neuroscience Methods, vol. 94, no. 1, pp. 105-119, Dec. 1999.

[15] J. Geweke, "Measurement of linear-dependence and feedback between multiple time series," Journal of the American Statistical Association, vol. 77, no. 378, pp. 304-313, Jun. 1982.

[16] J. Geweke, "Measures of conditional linear dependence and feedback between time series," J Am Stat Assoc, vol. 79, no. 388, pp. 907-915, Dec. 1984

[17] J. J. Ginter, K. J. Blinowska, M. Kaminski, P. J. Durka, G. Pfurtscheller, and C. Neuper, "Propagation of EEG activity in the beta and gamma band during movement imagery in humans," Methods Inf Med, vol. 44, no. 1, pp. 106-113, 2005.

[18] D. W. Gow, C. J. Keller, E. Eskandar, N. Meng, and S. S. Cash, "Parallel versus serial processing dependencies in the perisylvian speech network: A Granger analysis of intracranial EEG data," Brain and Language, vol. 110, no. 1, pp. 43-48, Jul. 2009.

[19] D. W. Gow, J. A. Segawa, S. Alfhors, and F. H. Lin, "Lexical influences on speech perception: A Granger causality analysis of MEG and EEG source estimates," Neuroimage, vol. 43, no. 3, pp. 614-623, Jul. 2008.

[20] C. W. J. Granger, "Investigating Causal Relations by Econometric Models and Cross-spectral Methods," Econometrica, vol. 37, no. 4, pp. 424-438, Aug. 1969.

[21] S. Guo, J. Wu, M. Ding, and J. Feng, "Uncovering interactions in the frequency domain,"Plos computational Biology, vol. 4, no. 5, e1000087:1-5, 2008
[22] W. Hesse, M. oller, M. Arnold, and B. Schack, "The use of time-variant EEG Granger causality for inspecting directed interdependencies of neural assemblies," J Neurosci Methods, vol. 124, no. 1, pp. 27-44, Mar. 2003.

[23] S. Hu, M. Stead, Q. Dai, and G. Worrell, "On the Recording Reference Contribution to EEG Correlation, Phase Synchorony, and Coherence,' IEEE Transactions on Systems, Man, and Cybernetics-Part B: Cybernetics, vol. 40, no. 5, pp. 1294-1304, Oct. 2010.

[24] D. Hwanga and A. Golby, "The brain basis for episodic memory: insights from functional MRI, intracranial EEG, and patients with epilepsy," Epilepsy \& Behavior, vol. 8, no. 1, pp. 115-126, Feb. 2006.

[25] S. Hu and H. Liang, "Causality analysis of neural connectivity: new tool and limitations of spectral Granger causality", Neurocomputing, accept for publication.

[26] M. J. Kahana, D. Seelig, and J. R. Madsen, "Theta returns", Curr Opin Neurobiol, vol. 11, no. 6, pp. 739-744, Dec. 2001.

[27] M. Kaminski, M. Ding, W. Truccolo-Filho, and S. L. Bressler, "Evaluating causal relations in neural systems: Granger causality, directed transfer function and statistical assessment of significance,' Biol Cybern, vol. 85, pp. 145-157, 2001.

[28] M. Kaminski and H. Liang, "Causal influence: advances in neurosignal analysis," critical reviews in biomedical engineering, vol. 33, no. 4, pp. 347-430, 2005.

[29] I. J. Kirk and J.C. Mackay, "The role of theta-range oscillations in synchronising and integrating activity in distributed mnemonic networks", Cortex vol. 39, no. 4, pp. 993-1008, Mar. 2003.

[30] A. Korzeniewska, C. M. Crainiceanu, R. Kus, P. J. Franaszczuk, and N. E. Cronel, "Dynamics of event-related causality in brain electrical activity," Human Brain Mapping, vol. 29, no. 10, pp. 1170-1192, Oct. 2008.

[31] A. Korzeniewska, S. Kasicki, M. Kaminski, and K. J. Blinowska, "Information flow between hippocampus and related structures during various types of rat behavior," J. Neurosci Methods, vol. 73, no. 1, pp. 49-60, Apr. 1997.

[32] A. Korzeniewska, M. Manczak, M. Kaminski, K. J. Blinowska, and S. Kasicki, "Determination of information flow direction among brain structures by a modified directed transfer function (dDTF) method," J. Neurosci Methods, vol. 125, no. 1, pp. 195-207, May 2003.

[33] R. Kus, M. Kaminski, and K. J. Blinowska, "Determination of EEG activity propagation: Pair-wise versus multichannel estimate," IEEE Trans Biomed Eng vol. 51, no. 9, pp. 1501-1510, Sep. 2004.

[34] H. Liang, M. Ding, R. Nakamura, S. L. Bressler, "Causal influences in primate cerebral cortex during visual pattern discrimination," $\mathrm{Neu}$ roreport, vol. 11, no. 13, pp. 2875-2880, Sep. 2000.

[35] H. Oya, P. W. F. Poon, J. F. Brugge, R. A. Reale, H. Kawasaki, and I O. Volkov, "Functional connections between auditory cortical fields in humans revealed by Granger causality analysis of intra-cranial evoked potentials to sounds: Comparison of two methods," Biosystems, vol. 89, pp. 198-207, 2007.

[36] M. Palu and M. Vejmelkay, "Directionality of coupling from bivariate time series: How to avoid false causalities and missed connections,' Phys. Rev. E, vol. 75, pp. 056211, 2007.

[37] S. Raghavachari, J. Lisman, M. Tully, J. Madsen, E. Bromfield, and M. Kahana, "Theta oscillations in human cortex during a working-memory task: Evidence for local generators," Journal of Neurophysiology, no. 95, no. 3, pp. 1630-1638, Mar. 2006.

[38] A. Roebroeck, E. Formisano, and R. Goebel, "Mapping directed influence over the brain using Granger causality and fMRI," Neuroimage vol. 25, no. 1, pp. 230-242, Mar. 2005.

[39] J. R. Sato, D. Y. Takahashi, S. M. Arcuri, K. Sameshima, P. A. Morettin, and L. A. Baccal,"Frequency domain connectivity identification: An application of partial directed coherence in fMRI," Human Brain Mapping, vol. 30, no. 2, pp. 452-461, Feb. 2009.

[40] B. Schelter, J. Timmer, and M. Eichler, "Assessing the strength of directed influences among neural signals using renormalized partial directed coherence," J. of Neuroscience Methods, vol. 179, no. 1, pp. 121-130, Apr. 2009.

[41] B. Schelter, M. Winterhalder, M. Eichler, M. Peifer, B. Hellwig, B. Guschlbauer, C. H. Lucking, R. Dahlhaus, and J. Timmer, "Testing for directed influences among neural signals using partial directed coherence," J. Neurosci Methods, vol. 152, pp. 210-219, Apr. 2005.

[42] A. Seghouane and S. Amari, "The AIC criterion and symmetrizing the KullbackCLeibler divergence," IEEE Trans. on Neural Networks, vol. 18, no. 1, pp. 97-106, Jan. 2007. 
[43] A. G. Siapas and M. A. Wilson, "Coordinated interactions between hippocampal ripples and cortical spindles during slow-wave sleep," Neuron, vol. 21, no. 5, pp. 1123-1128, 1998.

[44] E. J. Speckmann and C. E. Elger, "Introduction to the neurophysiological basis of the EEG and DC potentials," in Electroencepphalography: Basic Priniciples, Clinical Applications, and Related Fields, 5th edition, edited by E. Niedermeyer and F. Lopes Da Silva, Lippincott Williams \& Wilkins, Nov. 2004.

[45] R. Sun, "A neural network model of causality," IEEE Trans. on Neural Networks, vol. 5, no. 4, pp. 604-611, Jul. 1994.

[46] Y. Tanokura and G. Kitagawa, "Power contribution analysis for multivariate time series with correlated noise sources," Adv. Appl. Stat. vol. 4, no. 1, pp. 65-95, 2004.

[47] N. Wiener, The Theory of Prediction. In E.F. Beckenbach, editor, Modern Mathematics for Engineers, Chap. 8. McGraw-Hill, New York, 1956.

[48] O. Yamashita, N. Sadato, T. Okada, and T. Ozaki, “ Evaluating frequency-wise directed connectivity of BOLD signals applying relative power contribution with the linear multivariate time-series models," Neuroimage, vol. 25, no. 2, pp. 478-490, Apr. 2005. 